A s i a $\mathrm{n}$ J o u r n a l of

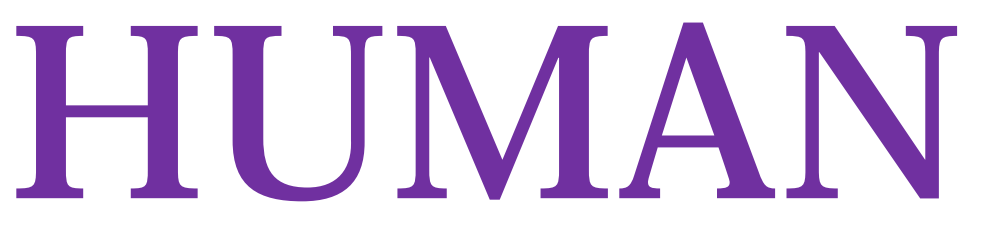

PRINTED 2020.0430 ISSN2188-059X

Published by ASIAN SOCIETY OF HUMAN SERVICES
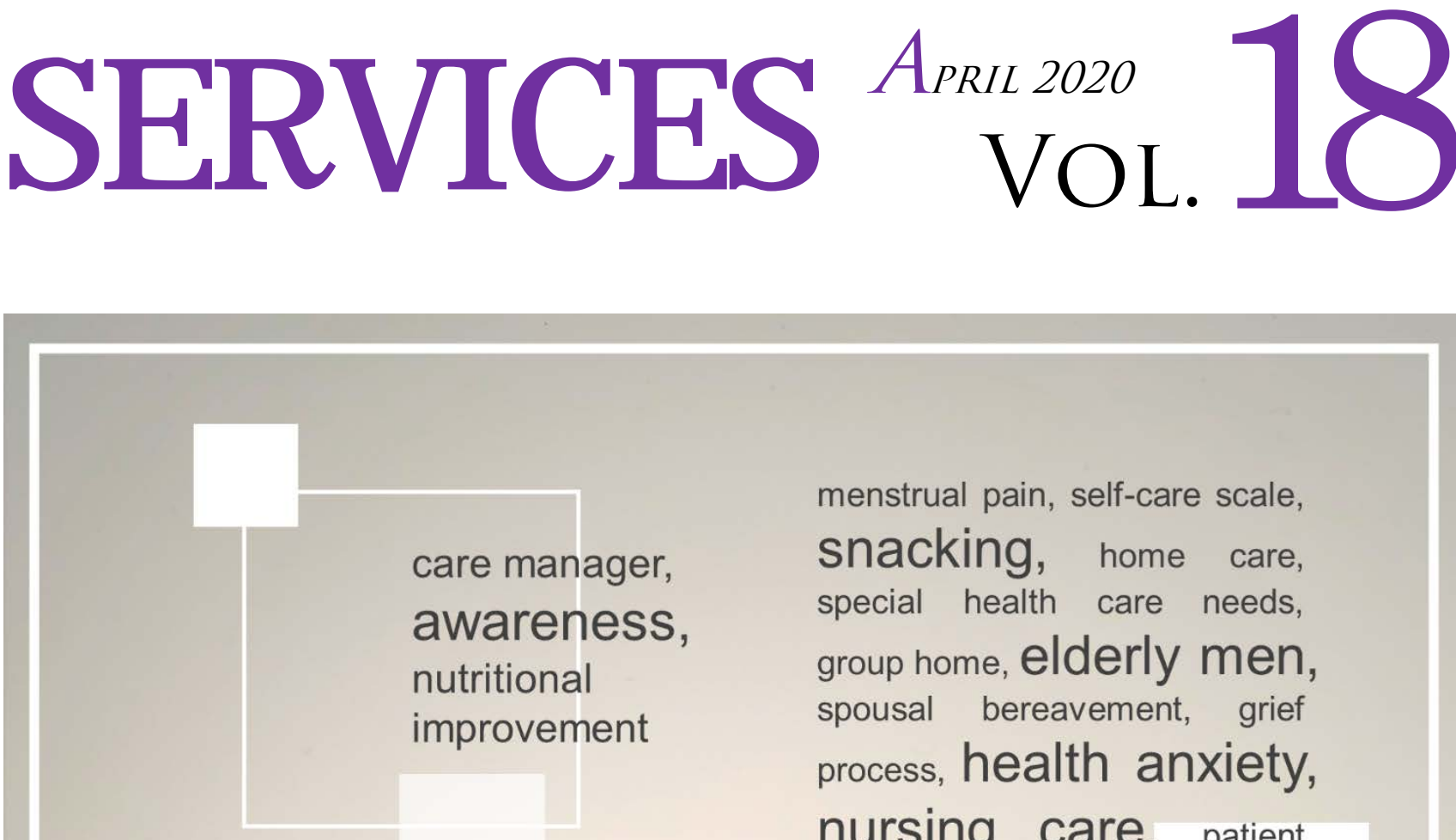

care manager, awareness, nutritional improvement

scale development, teacher training, home health nursing, instructors, down syndrome, verb, reflecting process, graduate students menstrual pain, self-care scale, snacking, home care, special health care needs, group home, elderly men, spousal bereavement, grief process, health anxiety, nursing care, patient acceptance system

Inclusive

education,

ICT utilization

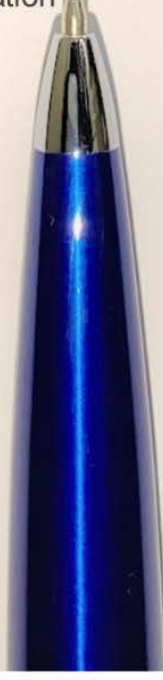




\title{
Services \\ Original Article \\ Structure of Care Managers' Approaches to and Awareness of "Nutritional Improvement" for Care-dependent Older People
}

\author{
Yuko FUJIO 1) Yoshiko ENOMOTO 1) Noriko OGAWA 1) \\ Kazutoshi FURUKAWA ${ }^{2)}$ Megumi KODAIRA ${ }^{3)}$ \\ 1) Faculty of Health Science and Nursing, Juntendo University, Japan \\ 2) Faculty of Human Life Design, Toyo University, Japan \\ 3) International University of Health and Welfare Granduate School, Japan
}

\begin{abstract}
To structure care managers' approaches (upright) to and awareness (oblique) of nutritional improvement for care-dependent older people, semi-structured interviews were conducted with 12 care managers in Japan. Concerning nutritional improvement for care-dependent older people, the care managers, managing older people with [mental and physical dysfunctions due to a decline in the nutritional status] and consequently [realizing the association between their nutritional status and mental and physical functions] developed dilemmas, while perceiving [distress due to problems care managers cannot resolve alone] as a result of [insufficient nutrition education through care manager training]. Although they perceived [distress due to problems care managers cannot resolve alone], they continued to adopt approaches, such as [devising measures for nutritional management from the perspective of a care manager] and [assessing nutritional status based on the living conditions and body weight]. However, the results

also revealed their dilemmas due to [difficulty in accurately assessing nutritional status], suggesting [the importance of performing nutritional management through collaboration with other professionals and services].
\end{abstract}

Received

November 26, 2019

Revised

January 21, 2020

Accepted

January 30, 2020

Published

April 30, 2020
$<$ Key-words $>^{-}$

structure, care manager, approaches to, awareness, nutritional improvement

yfujio@juntendo.ac.jp (Yuko FUJIO; Japan) Asian J Human Services, 2020, 18:1-17. C 2020 Asian Society of Human Services 


\section{Introduction}

Malnutrition is a major factor for older people requiring care. The revision of the Long-Term Care Insurance Act in 2006 included care prevention and preventing the level of care need from becoming higher, and nutrition management was introduced as the mainstay of the revision. However, according to the "Research on Comprehensive Evaluation and Analysis regarding the Effects of Care Prevention Programs" in 2008, among some older people or those requiring support who may continue requiring care after the initiation of care prevention programs, individuals requiring nutrition improvement account for approximately $30 \%$ of older people (Tsuji, Ueda, Okubo, et al., 2009). In addition, according to the "Survey Report about Understanding the Eating Condition and Nutritional conditions of Patients Receiving Home-Based Care" in 2012, approximately $30 \%$ of older people receiving home-based care suffer malnutrition based on MNA-SF (Mini Nutritional Assessment-Short Form) and BMI values (Body Mass Index) (National Center for Geriatrics and Gerontology, 2012). Thus, as issues regarding: 1) older people starting to require care and 2) the level of care need becoming higher, malnutrition has yet to be resolved.

This may be attributable to the absence of systems whereby identifying the risk of malnutrition among care service users (analysis) leads to care approaches that facilitate nutrition improvement (problem-solving). Regarding the identification of such a risk, in 2014, researchers conducted an awareness survey concerning the nutritional conditions of older people requiring care involving nursing care insurance service workers, and revealed that both home-based and facility workers were hardly aware of BMI values and Alb levels, which are indices of nutritional conditions (Fujio \& Kodaira, 2014). These results suggested that information-gathering and -sharing for identifying the risk of malnutrition were not standardized. In 2015, a survey was conducted to assess the nutritional conditions and mental/physical function of facility service users and at-home older people requiring care who utilized nursing care insurance services. As a result, correlations were noted between Alb levels and the following 4 factors: the BMI values, dietary habits, dietary intake, and locomotion ability. This indicated that these 4 factors may be predictors of Alb levels (Fujio, Ogawa, Inoue, et al., 2016). In 2016, a survey was conducted to assess the nutritional conditions and mental/physical function of older housing facility service users, which were not covered by nursing care insurance. In another survey conducted in the same year, we focused on the contents of information collected when creating care plans for nutrition improvement in long-term care insurance services. Alb levels were the least frequently collected content of information, followed by $\mathrm{BMI}$ and nutritional index values. The frequency of collecting nutritional index values was correlated with the number of service users, type of service, type of profession (basic qualifications), and experience of receiving nutrition education. The results indicated the necessity of considering the type of service, type of profession, and experience of receiving 
nutrition education as a challenge in creating care plans to improve older people's nutritional conditions (Fujio, Kurokawa, Furukawa, et al., 2018).

In a previous study examining the status of using information and communication technology (ICT) in the process of creating care plans, the use rate was generally high, but it was also suggested that ICT did not function as a problem-solving tool to link assessment and care plans and share the latter with service providers (Fujio, Enomoto, Furukawa, et al., 2019).

Therefore, the present study aimed to clarify the structure of care managers' approaches to and awareness of nutritional improvement for care-dependent older people. The clarification of approaches adopted by and awareness among care managers, who influence nutritional improvement for care-dependent older people, may provide a basis for standardizing care approaches to improve these people's nutritional status.

\section{Subjects and Methods}

\section{Study and Procedures}

1) Study Design

Qualitative inductive study design (Interview survey semi-structured interview method)

\section{2) Study Period}

Between October 1, 2017, and March 31, 2018

\section{3) Subjects}

Care managers providing nursing-care insurance services

\section{4) Study Items}

Basic attributes: age, sex, type of profession, years of experience, number of caseworks.

Interview method: An about 30-minute semi-structured interview session using an interview guide was held for each care manager.

Study items: The following questions were presented during each session: 1) Do you collect information regarding Alb levels and BMI values as nutritional indices for the creation of care plans?, 2) If you answered "No" for 1), how do you assess each user's nutritional status?, 3) If you answered "Yes" for 1), what institutions/types of profession do you collect such information from?, 4) Do you exchange information regarding the nutritional status with service providers?, 5) If you answered "Yes" for 4), what measures do you adopt for such an exchange? Please describe them in detail, 6) If you answered "No" for 4), what is the reason for not exchanging?, 7) Have you ever encountered cases where the user's level of mental and physical independence decreased due to a worsened 
nutritional status? If you have, please describe these cases in detail. 8) What institutions/types of profession do you consult with when users' levels of mental and physical independence decrease?, 9) Do you think there is an association between users' levels of mental and physical independence and nutritional status? If you answered "Yes"/"No", please describe the reason for answering so, 10) Have you ever received education regarding the relationship between care dependency/its progression and nutritional improvement through training or other activities? If you have, please describe the details of such education, and 11) Please let us know your thoughts on nutritional improvement for care-dependent older people in a free-description style.

\section{5) Ethical Considerations}

This study was conducted with the approval of the Ethics Committee of the Faculty of Health Science and Nursing, Juntendo University (approval number: 29-07). The study facilities and subjects were previously provided with written and oral explanations of the study objective, methods, voluntary cooperation, participants' right to withdraw at any time, and measures to ensure anonymity to obtain their consent.

\section{Data Collection}

Candidate facilities were selected using the opportunistic sampling method, and 6 home care support facilities located in the areas where the principal investigator and co-investigator were living were involved, asking their managers to introduce 1 care manager certified as a medical professional and 1 as a welfare professional.

\section{Data Analysis}

The interview data were organized as narrative records, which were carefully read and divided into minimum paragraphs with semantic contents as units for analysis. These units were encoded, focusing on care managers' approaches to and awareness of nutritional improvement, and classified into categories based on similarities with enhanced abstractness after careful deliberation on the data and codes to determine the characteristics and names of these categories. To enhance their validity, repeated discussions were held between the principal investigator and co-investigator. Additionally, word frequency analysis, dependency analysis, and correspondence analysis based on bubble charts were performed using Text Mining Studio Ver. 6.1 to confirm the validity of these categories by examining the relationships among the categories and between them and words. The relationships among the categories were further examined for structuring by examining similarities and differences among them. 


\section{Results}

\section{Basic Attributes (Table 1)}

The interviewees were 12 care managers belonging to 6 home care support facilities with the following basic qualifications: nurse: 4, pharmacist: 1, registered dietician: 1, care worker: 5 , and grade-2 helper: 1 . There were $4(33.3 \%)$ males and $8(66.7 \%)$ females in their thirties to sixties, and those in their fifties were the largest age group. The mean length of care manager experience was $9.67 \pm 4.73$ years, ranging from 3 to 17 years. The mean number of care plans created was $34.08 \pm 8.68$, ranging from 13 to 48 . The mean duration of an interview session was $19 \pm 6.53$ minutes, ranging from 10 to 31 minutes.

$<$ Table $1>$ Basic attributes

\begin{tabular}{ccccccc}
\hline & $\begin{array}{c}\text { Type of } \\
\text { profession }\end{array}$ & Sex & Age & $\begin{array}{c}\text { Interview time } \\
\text { (minutes) }\end{array}$ & $\begin{array}{c}\text { Years of } \\
\text { experience } \\
\text { (years) }\end{array}$ & $\begin{array}{c}\text { Number of } \\
\text { caseworks } \\
\text { (case) }\end{array}$ \\
\hline 1 & Nurse & Female & $50 \mathrm{~s}$ & 16 & 6 & 30 \\
2 & Nurse & Female & $60 \mathrm{~s}$ & 15 & 15 & 34 \\
3 & Nurse & Female & $60 \mathrm{~s}$ & 10 & 9 & 13 \\
4 & Pharmacist & Female & $50 \mathrm{~s}$ & 12 & 15 & 32 \\
5 & Nurse & Male & $50 \mathrm{~s}$ & 17 & 17 & 39 \\
6 & Nutritionist & Female & $40 \mathrm{~s}$ & 23 & 6 & 48 \\
7 & Care worker & Female & $40 \mathrm{~s}$ & 18 & 4 & 30 \\
8 & Care worker & Female & $50 \mathrm{~s}$ & 19 & 9 & 30 \\
9 & Care worker & Male & $40 \mathrm{~s}$ & 17 & 8 & 40 \\
10 & Care worker & Male & $40 \mathrm{~s}$ & 31 & 15 & 42 \\
11 & Care worker & Female & $50 \mathrm{~s}$ & 31 & 9 & 36 \\
12 & Care worker & Male & $30 \mathrm{~s}$ & 19 & 3 & $34.08 \pm 8.62$ \\
\hline \multicolumn{7}{c}{ Ave. } \\
\hline
\end{tabular}

\section{Categorization of the interview data (Table 2)}

The interview data were classified into 326 codes (\{\}), 33 sub-categories $(<>)$, and 8 categories ([ ]). In the following paragraphs, categories/sub-categories are listed from those with a larger number of codes.

[The importance of performing nutritional management through collaboration with other professionals and services]

\{Confirming day service users' dietary intakes and changes in them\} and \{collecting information to identify marked changes in the body weight most frequently from day services\} were classified into <performing nutritional management through collaboration 
with day services>. \{Helper intervention as part of home-visit care enabling users to take 3 meals a day\} and \{confirmation and consultation with care managers dispatched at the request of helper service facilities\} were classified into <performing nutritional management through collaboration with helpers $>$. \{Visiting nurses who are the easiest to collaborate with\} and the ease of collecting information regarding blood Alb and glucose levels from hospitals providing home-visit nursing services\} were classified into <performing nutritional management through collaboration with visiting nurses>. Creating a team to support users and resolve their problems care managers cannot resolve alone\} and \{relying on service providers' specialized perspectives\} were classified into <creating a team for collaboration>. \{Observing users, including their family relationships, through teamwork\} and \{asking users losing body weight or their families about the former's conditions\} were classified into < performing nutritional management with cooperation from users and their families>. \{Collecting opinions from attending doctors\} and \{active contact from home care doctors\} were classified into <performing nutritional management through collaboration with attending doctors $>$. \{Collecting information from short stay services\} and \{Confirming the dietary status recorded using check sheets in short stay services\} were classified into <performing nutritional management through collaboration with those engaged in short stay services $>$. These sub-categories were finally summarized into [the importance of performing nutritional management through collaboration with other professionals and services], consisting of 76 codes, which was the largest number.

\section{[Mental and physical dysfunctions due to a decline in the nutritional status]}

\{A loss of motivation to eat due to eating alone day after day\} and \{significant differences in the dietary pattern and nutritional status between older people living with other family members and those living alone\} were classified into < reduced dietary intake due to the influences of a solitary life and living environment>. \{An increased incidence of falls possibly associated with insufficient nutrition and \{the development of dementia symptoms after becoming bedridden due to a worsened nutritional status\} were classified into <declined functioning due to a decline in the nutritional status $>$. \{The necessity of admission to a care facility or hospital due to a decline in the nutritional status\} and \{difficulty in maintaining a desired home life due to reduced dietary intake\} were classified into < difficulty in leading a desired life due to a decline in the nutritional status $>$. \{An insufficient understanding of malnutrition among other family members\} and \{giving up trying to eat due to a loss of appetite in many cases\} were classified into <insufficient nutrition resulting from users' and their families' insufficient understanding $>$. \{Reduced dietary intake due to swallowing dysfunction\} and \{negative attitudes resulting in poor eating habits\} were classified into <insufficient nutrition due to mental/physical dysfunction or a disease>. \{Directly influencing users' levels of independence and caregiving burdens\} and \{difficulty in continuing to use day services\} were classified into <increased caregiving burdens due to a decline in the nutritional 
status>. These sub-categories were finally summarized into [mental and physical dysfunctions due to a decline in the nutritional status], consisting of 45 codes.

[Assessing the nutritional status based on the living conditions and body weight]

\{Asking about activities performed each day to assess the nutritional status, as assessment based only on diets is insufficient\} and \{observing kitchens and refrigerators to clarify cooking habits\}, and \{identifying the places each user goes for shopping and asking about his/her status\} were classified into <asking about activities performed each day and living conditions to assess the nutritional status $>$. \{Directly observing users have meals in some cases\} and \{confirming eating styles as part of assessment in all cases\} were classified into <assessing the nutritional status based on dietary intake>. The necessity of observation based on the body weight\}, \{measuring the body weight of users with a loss of appetite\} were classified into <assessing the nutritional status based on the body weight>. These sub-categories were finally summarized into [assessing the nutritional status based on the living conditions and body weight], consisting of 44 codes. [Realizing the association between the nutritional status and mental and physical functions]

\{Considering diets as a source of life\}, \{realizing the strong demand for nutritional management\}, and \{becoming aware of the importance of nutritional management through experience\} were classified into <placing importance on nutrition>. \{A rapid decline in physical functions due to insufficient nutrition\} and \{the rapid development of a bedridden condition after fracture and pressure ulcers due to a poor nutritional status\} were classified into < declines in mental and physical functions due to a poor nutritional status $>$. \{Perceiving the marked influence of the nutritional status on older people's independence\} and \{clearly perceiving the physical and mental influences of the nutritional status\} were classified into <perceiving the association between users' nutritional status and mental/physical independence>. These sub-categories were finally summarized into [realizing the association between nutritional status and mental and physical functions], consisting of 43 codes.

[Devising measures for nutritional management from the perspective of a care manager]

\{Considering physical fitness and environmental factors to promote dietary intake\} and \{improving the nutritional balance while considering users' food preferences\} were classified into <devising feasible measures as a care manager $>$. \{Anticipating possible situations due to missing appropriate timings and \{intervening with the minimum possible burden on families\} were classified into <intervening without missing the timing $>$. \{Incorporating the nutritional status into care plans and continuously observing it when it is shown to be poor on assessment\} and \{the ease of collecting information regarding the nutritional status from care providers after incorporating it into care plans\} were classified into <incorporating the nutritional status into care plans and monitoring $>$. These sub-categories were finally summarized into [devising measures for nutritional management from the perspective of a care manager], consisting of 36 codes. 


\section{[Distress due to problems care managers cannot resolve alone]}

\{A lack of expertise\} and the necessity of new social resources for nutritional improvement\} were classified into <seeking support from other professionals $>$. \{Difficulty in changing users' eating habits at the age of 80 or 90 , as care management is based on their previous diets\} and the uncertainty of adherence to the advised dietary requirements\} were classified into <limitations of care management>. \{Unestablished problem-solving processes\} and \{having provided similar guidance for problem-solving over the last 10 years\} were classified into <insufficient knowledge of methodologies for nutritional improvement>. \{Not perceiving the importance of nutritional management\} and \{considering nutritional management as out of the scope of care manager services despite a high demand for it $\}$ were classified into <care managers' poor perception of the necessity of nutritional management $>$. These sub-categories were finally summarized into [distress due to problems care managers cannot resolve alone], consisting of 35 codes.

\section{[Insufficient nutrition education through care manager training]}

Insufficient opportunities to learn about nutrition despite the availability of various practical training seminars for care managers\} and \{care manager training not addressing care management for people with malnutrition\} were classified into $<$ having never received nutrition-related education>. \{Having participated in training seminars held by medical professionals, such as registered dieticians and nurses\} and \{having learned about oral exercise and oral care through training seminars held by visiting dentists\} were classified into <having received nutrition-related education through training seminars not targeting care managers $>$. \{Desiring to participate in training for nutritional improvement\} was classified into <desiring to participate in care manager training regarding nutrition>. These sub-categories were finally summarized into [insufficient nutrition education through care manager training], consisting of 25 codes. [Difficulty in accurately assessing the nutritional status]

\{Difficulty in asking further questions to family members who state that the patient is sufficiently eating\} and \{the necessity of paying attention to possible weight loss even when other family members state that the patient is sufficiently eating\} were classified into < difficulty in assessing the nutritional status based only on information from families>. \{Difficulty in clarifying whether the user is refusing to eat, or his/her physical condition does not allow eating\} and \{difficulty in clarifying the types of food consumed and level of their consumption in the case of dementia\} were classified into < difficulty in assessing users' nutritional status based only on their conditions $>$. \{Difficulty in collecting information regarding Alb levels from attending doctors\} and \{hesitating to directly make telephone calls to doctors from a care manager\} were classified into $<$ difficulty in confirming the status by inquiring of attending doctors $>$. \{Difficulty in clarifying Alb levels\} and \{perceiving difficulty in collecting truly objective information\} were classified into < difficulty in clarifying Alb levels and evaluating other parameter 
values>. These sub-categories were finally summarized into [difficulty in accurately assessing the nutritional status], consisting of 22 codes. Table 2 lists all categories and sub-categories.

$<$ Table $2>$ Categorize interview results $(\mathrm{n}=326)$

\begin{tabular}{l}
\hline \multicolumn{1}{c}{ Categories } \\
\hline \\
The importance of performing \\
nutritional management \\
through collaboration with \\
other professionals and services \\
(76)
\end{tabular}
Codes

Performing nutritional management through collaboration with day services

Performing nutritional management through collaboration with helpers

Performing nutritional management through collaboration with visiting nurses

Creating a team for collaboration

Performing nutritional management with cooperation from users and their families

Performing nutritional management through collaboration with

Mental and physical dysfunctions due to a decline in the nutritional status (45)
Assessing the nutritional status based on the living conditions and body weight (44)

Realizing the association between the nutritional status and mental and physical functions (43)

Devising measures for nutritional management from the perspective of a care manager (36)

Distress due to problems care managers cannot resolve alone (35)

Insufficient nutrition education through care manager training (25)

Difficulty in accurately assessing nutritional status (22) attending doctors

Performing nutritional management through collaboration with those engaged in short stay services

Reduced dietary intake due to the influences of a solitary life and living environment

Declined functioning due to a decline in nutritional status

Difficulty in leading a desired life due to a decline in nutritional

status

Insufficient nutrition resulting from users' and their families' insufficient understanding

Insufficient nutrition due to mental/physical dysfunction or a disease

Increased caregiving burdens due to a decline in nutritional status

Asking about activities performed each day and living conditions to assess the nutritional status

Assessing the nutritional status based on dietary intake Assessing the nutritional status based on body weight

Placing importance on nutrition

Declines in mental and physical functions due to a poor nutritional status

Perceiving the association between users' nutritional status and mental/physical independence

Devising feasible measures as a care manager 16

Intervening without missing the timing

Incorporating the nutritional status into care plans and monitoring

Seeking support from other professionals

Limitations of care management

\section{5}

2

15

Insufficient knowledge of methodologies for nutritional improvement

Care managers' poor perception of the necessity of nutritional management

Having never received nutrition-related education

Having received nutrition-related education through training seminars not targeting care managers

Desiring to participate in care manager training regarding nutrition

Difficulty in assessing the nutritional status based only on information from families

Difficulty in assessing users' nutritional status based only on their condition

Difficulty in confirming the status by inquiring of attending doctors

Difficulty in clarifying Alb levels and evaluating other parameter values 
3. Analysis of the relationships among the categories and between them and frequent words (Figure 1)

The care managers' narratives were analyzed using Text Mining Studio Ver. 6.1 to examine the relationships among the categories and between them and relevant words. First, each word (morpheme)'s frequency of appearing was confirmed. The following words ranked among the top 20 through word frequency analysis: "eat", "meal", "ask", "nutrition", "body weight", "care manager", "family", "eat + not", "day services", "nutritional status", "create”, "confirm”, "observe", "take/collect”, "intervene”, "very", "many", "the user", "helper", "independence", and "information". These frequent words were included in fragmentated codes.

Subsequently, dependency analysis was performed to confirm morpheme-morpheme syntactical relationships. The 20 most frequent relationships were as follows: [independence - relationships], [care manager -training], [connections - create], [nutrients - take in + not], [body weight - measure], [3 meals - diet], [lunch - take], [care manager - planning], [day services - ask], [helper - collaborate], [helper - intervene], [opinion - ask], [nutrients - take in], [nutritional status - poor], [family - confirm], [family - eat], [relationships - create], [face - observe + can], [difficulty - perceive]. These words were contained in the relevant codes or sub-categories and categories created from them.

Furthermore, the levels of correlation among the created categories and between them and the frequent words were confirmed using bubble charts for correspondence analysis. These charts examine correlations based on the distances between mapped attributes and words. In the present study, analysis was performed, with the categories created from the interview data replacing attributes. On examining the relationships among the categories, [assessing the nutritional status based on the living conditions and body weight] was very close to and the most strongly correlated with the largest category: [the importance of performing nutritional management through collaboration with other professionals and services]. [Difficulty in accurately assessing the nutritional status] was the second closest to it, followed by [mental and physical dysfunctions due to a decline in the nutritional status] and then [devising measures for nutritional management from the perspective of a care manager]. In contrast, [insufficient nutrition education through care manager training] was distant, but this category was close to and strongly correlated with [distress due to problems care managers cannot resolve alone]. [Devising measures for nutritional management from the perspective of a care manager] and [realizing the association between nutritional status and mental and physical functions] were close to and strongly correlated with [distress due to problems care managers cannot resolve alone].

As for the relationships between the categories and frequent words, [the importance of performing nutritional management through collaboration with other professionals and services] was very close to and strongly correlated with "day services", "create", "information", "confirm", and "helper". These words were contained in the codes 
constituting [the importance of performing nutritional management through collaboration with other professionals and services], including: \{collecting information to identify marked changes in the body weight most frequently from day services\}, \{helper intervention as part of home-visit care enabling users to take 3 meals a day\}, \{creating a team to support users and resolve their problems care managers cannot resolve alone\}, and \{asking users losing body weight or their families about the former's conditions\}.

[Assessing the nutritional status based on the living conditions and body weight] was very close to and strongly correlated with "body weight", "observe", and "ask". These words were contained in the codes constituting [assessing the nutritional status based on the living conditions and body weight], including: [the necessity of observation based on the body weight\} and \{asking about activities performed each day to assess the nutritional status, as assessment based only on diet is insufficient\}.

[Difficulty in accurately assessing the nutritional status] was close to and strongly correlated with "the user" and "family" and "collect" and "eat". These words were contained in the codes constituting [difficulty in accurately assessing the nutritional status], including: \{difficulty in clarifying whether the user is refusing to eat, or his/her physical condition does not allow eating\}, \{difficulty in asking further questions to family members who state that the patient is sufficiently eating\}, and \{perceiving difficulty in collecting truly objective information\}.

[Mental and physical dysfunctions due to a decline in the nutritional status] was close to and strongly correlated with "eat + not", "many", and "meals". These words were contained in the codes constituting [mental and physical dysfunctions due to a decline in the nutritional status], including: \{a loss of motivation to eat due to eating alone day after day\}, \{giving up trying to eat due to a loss of appetite in many cases\}, and \{reduced dietary intake due to swallowing dysfunction\}.

[Devising measures for nutritional management from the perspective of a care manager] was close to and strongly correlated with "intervene". This word was contained in the codes constituting [devising measures for nutritional management from the perspective of a care manager], including: \{intervening with the minimum possible burden on families\} and \{incorporating the nutritional status into care plans and continuously observing it when it is shown to be poor on assessment\}.

"Care manager" and "nutrition" were placed between [insufficient nutrition education through care manager training] and [distress due to problems care managers cannot resolve alone], revealing strong correlations. These words were contained in the code constituting [insufficient nutrition education through care manager training], including \{care manager training not addressing care management for people with malnutrition\}, and that constituting [distress due to problems care managers cannot resolve alone], including \{considering nutritional management as out of the scope of care manager services despite a high demand for it\}.

[Realizing the association between nutritional status and mental and physical 
functions] was close to and strongly correlated with "independence", "nutritional status", and "very". These words were contained in the codes constituting, [realizing the association between the nutritional status and mental and physical functions], including: \{perceiving the marked influence of the nutritional status on older people's independence\} and the rapid development of a bedridden condition after fracture and pressure ulcers due to a poor nutritional status\}. Figure 1 shows the results of correspondence analysis using bubble charts.

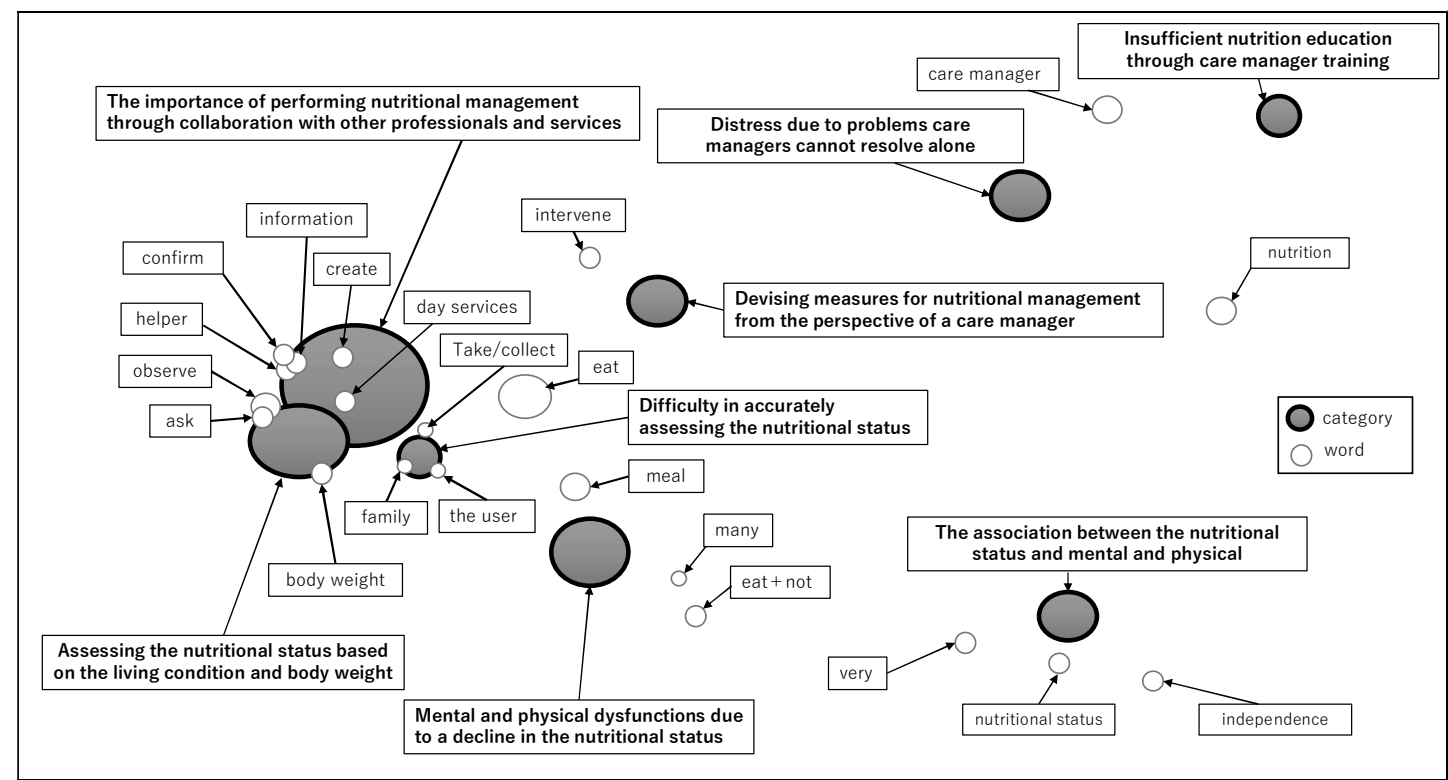

$<$ Figure $1>$ Correspondence bubble analysis results between categories and frequent words

4. Structuring of care managers' approaches to and awareness of nutritional improvement for care-dependent older people (Figure 2)

Based on the created categories, care managers' approaches to and awareness of nutritional improvement for care-dependent older people were structured. In the following paragraphs, approach- and awareness-related categories are shown in upright and oblique letters, respectively. Concerning nutritional improvement for care-dependent older people, the care managers managing older people with [mental and physical dysfunctions due to a decline in the nutritional status] and consequently [realizing the association between nutritional status and mental and physical functions] developed dilemmas, while perceiving [distress due to problems care managers cannot resolve alone] as a result of [insufficient nutrition education through care manager training]. Although they perceived [distress due to problems care managers cannot resolve alone], they continued to adopt approaches, such as [devising measures for nutritional management from the perspective of a care manager] and [assessing the nutritional 
status based on the living conditions and body weight]. However, the results also revealed their dilemmas due to [difficulty in accurately assessing the nutritional status], suggesting Ithe importance of performing nutritional management through collaboration with other professionals and services]. Figure 2 illustrates the structure of care managers' approaches to and awareness of nutritional improvement for care-dependent older people.

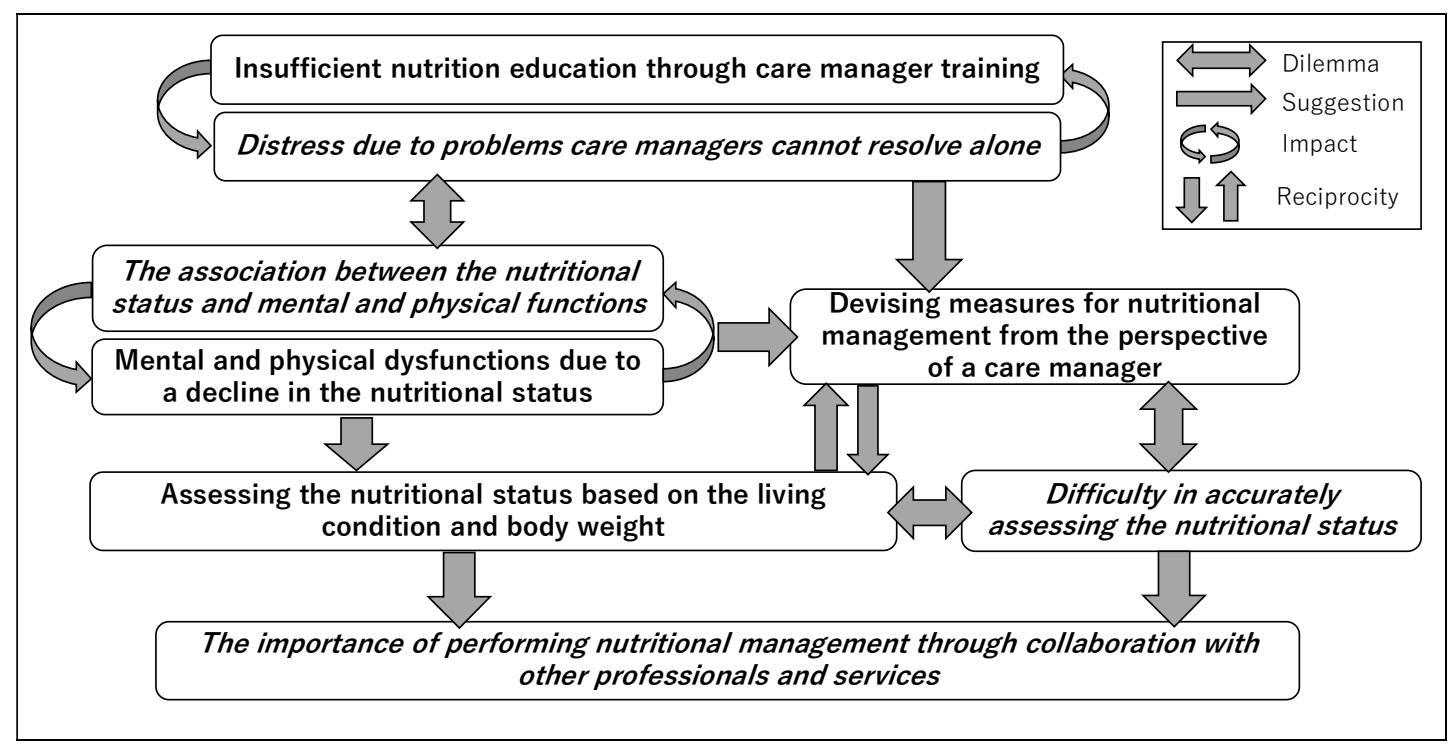

$<$ Figure 2> Structure of the care manager's approaches to and awareness of "nutritional improvement" for the older people

\section{Discussion}

Among the 12 care managers interviewed, there were 6 medical and 6 welfare professionals. Those in their fifties were the largest group, and the mean length of care manager experience was nearly 10 years. Thus, the majority of the interviewees had managed a large number of cases as care managers.

The largest category [the importance of performing nutritional management through collaboration with other professionals and services], consisting of 76 codes, represents care managers' awareness. Background factors associated with the highest frequency of this category being mentioned by the care managers are discussed while considering its relationships with the other categories, as follows: [The importance of performing nutritional management through collaboration with other professionals and services] was strongly correlated with [assessing the nutritional status based on the living conditions and body weight]. For the care managers, [assessing the nutritional status based on the living conditions and body weight] for care-dependent older people, to suggest [the importance of performing nutritional management through collaboration 
with other professionals and services], experiencing the management of those with [mental and physical dysfunctions due to a decline in the nutritional status] as an approach and [realizing the association between nutritional status and mental and physical functions] as a state of awareness may have been important. The correlation and mutual influence between these 2 categories were strong. To address such a situation, the care managers continued to adopt approaches, such as [devising measures for nutritional management from the perspective of a care manager], possibly leading to [assessing the nutritional status based on the living conditions and body weight]. These 2 categories were also strongly correlated, suggesting interactions between them. At the same time, the care managers may have developed a dilemma, adopting the 2 approaches, [assessing the nutritional status based on the living conditions and body weight] and [devising measures for nutritional management from the perspective of a care manager], while being aware of the [difficulty in accurately assessing the nutritional status]. [Insufficient nutrition education through care manager training] and [distress due to problems care managers cannot resolve alone] were described as factors associated with such awareness and approaches, and the correlation and mutual influence between these were also strong. The care managers may have experienced another dilemma due to [distress due to problems care managers cannot resolve alone] and [realizing the association between nutritional status and mental and physical functions]. However, [distress due to problems care managers cannot resolve alone] may have put them into [devising measures for nutritional management from the perspective of a care manager], and [devising measures for nutritional management from the perspective of a care manager] may have paved the way for [assessing the nutritional status based on the living conditions and body weight]. The care managers' awareness of [the importance of performing nutritional management through collaboration with other professionals and services] may have been promoted by realizing the [difficulty in accurately assessing the nutritional status] and consequently experiencing a dilemma. The structure shown in Figure 2 outlines these approaches adopted by and awareness among care managers to improve care-dependent older people's nutritional status.

Nutritional improvement for care-dependent older people is a measure to prevent care dependency and its progression or a methodology to support the independence of these people, adopted with the revision of the Long-Term Care Insurance Act in 2006. Skills needed by care managers to provide such support are classified into 5 domains: counseling skills, knowledge and skills required for care management, trust-based relationships, personality changes through communication, and teamwork (Makino, 2013). Among the 5 domains, the status of knowledge and skills required for care management was described as \{care manager training not addressing care management for people with malnutrition\} by care managers in the present study, revealing [insufficient nutrition education through care manager training]. In the author's previous study, nutrition education was a challenge in creating care plans for nutritional 
improvement (Fujio, Kurokawa, Furukawa, et al., 2018). The care managers' narratives, revealing insufficient nutrition education, are consistent with this. Some researchers also note that few people understand why a poor nutritional status among older people is problematic (Kato, 2014), confirming that the provision of sufficient nutrition education for care managers is an important challenge. The Guidelines on Integrated Care for Older People (ICOPE) published by the World Health Organization (WHO) in 2017 focus on malnutrition as an area to be addressed through integrated care for older people (WHO, 2017).

Insufficient nutrition education was described as \{unestablished problem-solving processes\} and \{a lack of expertise\} by the care managers, perceiving [distress due to problems care managers cannot resolve alone]. On the other hand, managing older people with [mental and physical dysfunctions due to a decline in the nutritional status], represented by \{the development of dementia symptoms after becoming bedridden due to a worsened nutritional status\}, and [realizing the association between nutritional status and mental and physical functions], they developed a dilemma, described as \{clearly perceiving the physical and mental influences of the nutritional status\}. They addressed such a situation by [devising measures for nutritional management from the perspective of a care manager], such as \{incorporating the nutritional status into care plans and continuously observing it when it is shown to be poor on assessment\}, and [assessing the nutritional status based on the living conditions and body weight] while considering [the necessity of observation based on the body weight\}. However, they also experienced a dilemma due to [difficulty in accurately assessing nutritional status], specifically \{difficulty in asking further questions to family members who state that the patient is sufficiently eating\}.

With regard to dilemmas experienced by care managers, communication is thought to resolve social dilemmas in socio-psychology (Norbert, \& Kaufman-Gilliland, 1994). Such communication may explain [the importance of performing nutritional management through collaboration with other professionals and services], noted as \{creating a team to support users and resolve their problems care managers cannot resolve alone\} by many care managers in the present study. Nutritional management through collaboration with other professionals and services may promote communication, and consequently resolve dilemmas experienced by care managers trying to improve care-dependent older people's nutritional status. Kira stated, "Long-term relationships and communication have different functions to resolve social dilemmas" (Kira, 2013), as "there is a long-term relationship, but there is no arena for communication" (Kira, 2013). The care managers mentioned collaboration with other professionals and services, not indicating shallow relationships, such as acquaintances, but possibly suggesting the importance of promoting communication as part of team approaches to improve care-dependent older people's nutritional status. In team approaches for care management, communication is regarded as an important tool to confirm common goals, share necessary information, 
and provide mutual support among team members (Shirasawa, Hashimoto \& Takeuchi, 2006). The structure of care managers' approaches to and awareness of nutritional improvement for care-dependent older people highlights the importance of communicating and collaborating with other professionals and services.

\section{Acknowledgment}

The authors wish to thank for care managers of the nursing care insurance service in Japan. This work was supported by JSPS KAKENHI Grant No. 16K12218 (Interprofessional Cooperation ICT Program Development aimed at "Nutrition Improvement").

\section{References}

1) Ichiro Tsuji, Koichiro Ueda, Ichiro Okubo, Yutaka Ono, Satoko Ohara, Shuichi Obuchi, et al. (2009) Comprehensive assessment and analysis of the effects of the Projects to Prevent the Need for Care, A Grant-in-Aid for Health Care Promotion Services for the Elderly (Promotion of Health and Health Care for the Elderly).

2) National Center for Geriatrics and Gerontology (2012) "Survey Report about Understanding the Eating Condition and Nutritional Status of Patients Receiving Home-Based Care" (Project for Geriatric Health Promotion, etc.).

3) Yuko Fujio \& Megumi Kodaira (2014) Care Service Staff's Awareness of the Management of Undernutrition in Japan, Asian Journal of Human Services, 7, 51-59. doi: 10. 14391/ajhs.7.51.

4) Yuko Fujio, Noriko Ogawa, Yoshiyuki Inoue, Megumi Kodaira \& Takahito Takeuchi (2016) Indices of Undernutrition in the Care-dependent Elderly. Asian Journal of Human Services, 10, 16-24. doi: 10. 14391/ajhs.10. 16.

5) Yuko Fujio, Yoshiko Kurokawa, Kazutoshi Furukawa, Megumi Kodaira \& Noriko Ogawa (2018) Survey to Assess Information-gathering During the Process of Designing Care Plans Regarding Nutrition Improvement in Nursing Care Insurance Services, Asian Journal of Human Services, 14, 11-23. doi: 10.14391/ajhs.14.11.

6) Yuko Fujio, Yoshiko Enomoto, Kazutoshi Furukawa, Megumi Kodaira \& Noriko Ogawa (2019) Interprofessional Cooperation ICT Program Development aimed at "Nutrition Improvement", Asian Journal of Human Services, 16, 45-57.

doi: 10.14391/ajhs.16.45. 
7) Kazuko Makino (2013) The research concerning the visualization of the factors which care managers should acquire in order to achieve a self-support of users. Graduate School of Health and Welfare Sciences, International University of Health and Welfare, doctoral thesis.

8) Masahiko Kato (2014) Nutrition Care for the Self-actualization of Older People Living at Home, Life Sciences. 1-10.

9) WHO Guidelines on Integrated Care for Older People (ICOPE). https://www.who.int/ageing/publications/guidelines-icope/en/

10) Kerr Lee Norbert \& Cynthia Marie Kaufman-Gilliland (1994) Communication. Commitment. And Cooperation in Social Dilemma, Journal of Personality and Social Psychology, 66 (3), 513-529. doi: 10.1037/0022-3514.66.3.513.

11) Yosuke Kira (2013) Why Does Communication Resolve Social Dilemmas? Equilibrium Refinement in Iterated N-person Prisoner's Dilemma, Sociological Theory and Methods, 28, 1, 107-124. doi: 10.11218/0jjams.28.107.

12) Kazumasa Shirasawa, Yasuko Hashimoto \& Takahito Takeuchi (2006) Lecture on Care Management 1: An Introduction to Care Management. Chuohoki Publishing CO., Ltd. 


\title{
ASIAN JOURNAL OF HUMAN SERVICES EDITORIAL BOARD
}

\author{
EDITOR-IN-CHIEF \\ Masahiro KOHZUKI Tohoku University (Japan) \\ EXECTIVE EDITORS \\ LEE, In Jae Hanshin Univerisity (Korea) \\ Satoru EBIHARA Toho University (Japan)
}

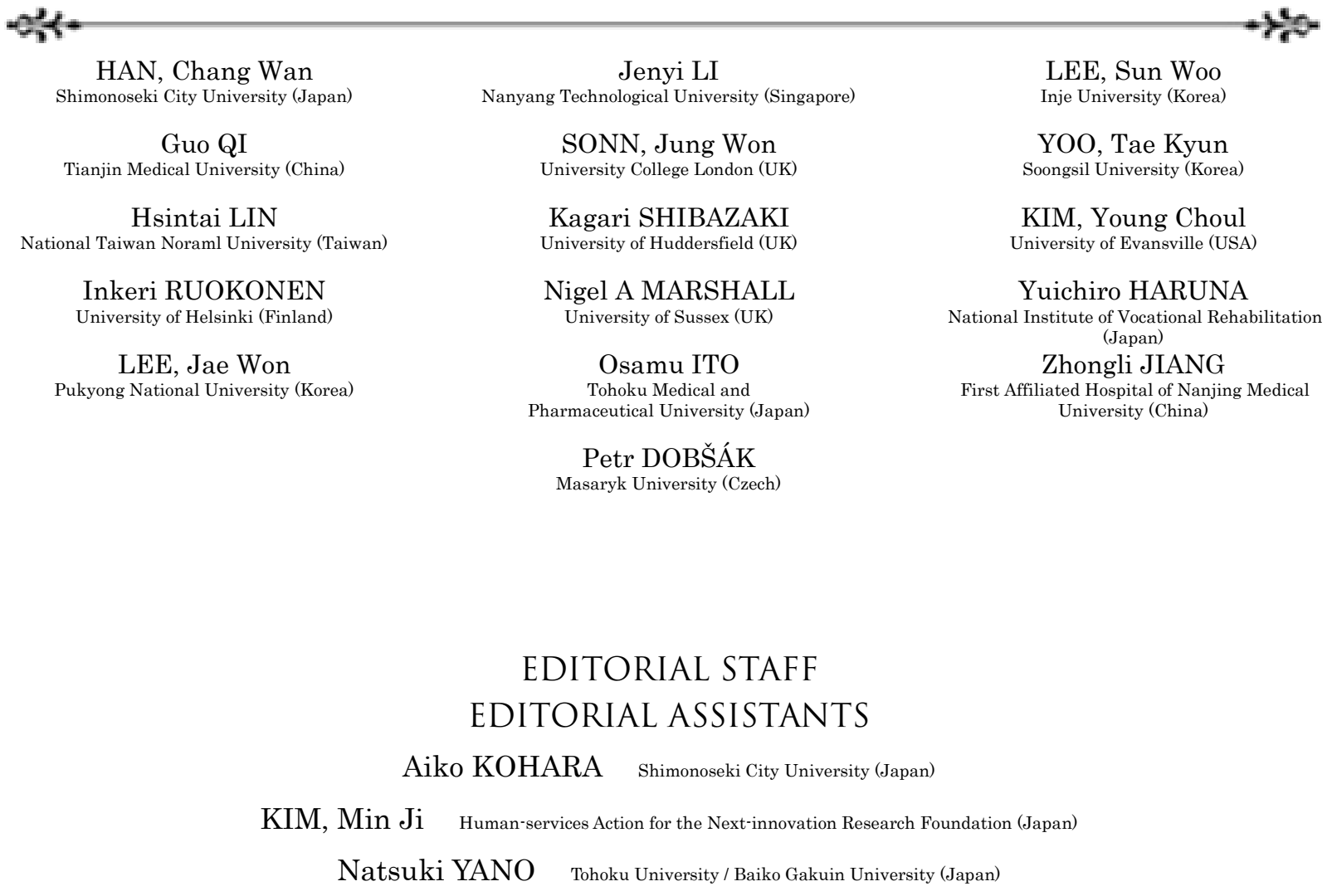

\section{ASIAN JOURNAL OF HUMAN SERVICES}

\section{VOL.18 April 2020}

(C) 2020 Asian Society of Human Services

$\begin{array}{ll}\text { Presidents } & \text { Masahiro KOHZUKI \& LEE, Sun Woo } \\ \text { Publisher | } & \text { Asian Society of Human Services } \\ & \text { \#1Floor Ohara Bill, 2-11-5, Takezaki-Town, Shimonoseki-City, Yamaguchi-Prefecture, 750-0025, Japan } \\ & \text { E-mail: ashs201091@gmail.com } \\ \text { Production } \mid & \text { Asian Society of Human Services Press } \\ & \text { \#1Floor Ohara Bill, 2-11-5, Takezaki-Town, Shimonoseki-City, Yamaguchi-Prefecture, 750-0025, Japan } \\ & \text { E-mail: ashs201091@gmail.com }\end{array}$




\section{ASIAN JOURNAL OF HUMAN SERVICES \\ VOL.18 April 2020}

\section{CONTENTS}

\section{ORIGINAL ARTICLES}

Structure of Care Managers' Approaches to and Awareness of "Nutritional Improvement" for Care-dependent Older People

Yuko FUJIO et al. p.1

A Multi-population Analysis of a Self-care Scale for Menstrual Pain:

Causal Relationships between Snacking and Menstrual Pain

Eriko YAMAMOTO p.18

Issues in Spousal Bereavement Support for Elderly Men in Japan

Makiko YAMAUCHI et al. p.33

A Fundamental Study on Health Anxiety in the Daily Life of Visually Impaired People Living in the Community and the Actual Situation of Patient Acceptance Systems at Medical Institutions

Keiko OMOTO p.48

Development of the Disability Awareness Program (DAP) scale:

Centering on the Content Validity Verification

Mamiko OTA et al. p.63

\section{SHORT PAPERS}

Medical and Care Collaboration between Nurse and Care-worker in a 'Kantaki' Setting: Time-Sampling Study

Yumi FUKUYAMA et al. p.74

Preliminary Study on Verb Acquisition Status and Related Factors in Children with Down Syndrome

Haruna OKAMIYA et al. p. 81

Experience of Reflecting Process in Graduate School for Clinical Psychologist

Norimasa ITAKURA et al. p.92

\section{CASE REPORTS / ACTIVITY REPORTS}

Analysis of Difficultiens faced by Home Health Nursing Practicum Instructors

Reiko HATAKEYAMA et al. p.100

UDL using ICT for Inclusive Learning; Learning Support for Students with

Diverse Learning Styles, Including Students who Need Special Support

Mitsuyo SHIMOJO et al. p.112

Published by

Asian Society of Human Services

Yamaguchi, Japan 